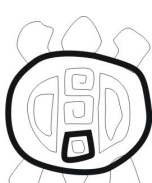

RMA

Traducción

Antropología Social

Hay algo curioso en la antropología: al tiempo que se vanagloria de tener una de las tradiciones más sólidas entre las ciencias sociales - en la cual se reconocen cronológicamente los mismos autores "clásicos", sea en Brasil, Estados Unidos, la India o Inglaterra - la disciplina abarca estilos bastante diferenciados, en la medida en que factores como el contexto de investigación, la orientación teórica, el momento socio-histórico y hasta la personalidad del investigador y el ethos de los investigados influencian el resultado obtenido. Esta característica, al mismo tiempo que puede ser apropiada positivamente como uno de los aspectos más ricos y complejos de la disciplina, por otro lado ofrece el peligro de, al no respetar el equilibro sutil entre teoría e investigación, transformarse en una situación en la cual existen tantas antropologías como antropólogos.

Esta tal vez sea la fuente de la situación problemática que la antropología ofrece potencialmente a las demás ciencias sociales y que Fábio Wanderley Reis apuntó, en 1988, cuando detectó cierta inspiración "antropológica" en los trabajos poco sofisticados de las ciencias sociales brasileras en la actualidad. ' Privilegiando lo "popular", el lector tenía que soportar "largas deposiciones en estado bruto de mujeres de la periferia urbana", una descripción que sirve como metáfora para muchos de los problemas que ocurren también dentro de la disciplina. Más recientemente, el autor denunció también cierto "conyunturalismo" y un "historicismo" como responsables por la ausencia de una mayor y deseable sofisticación teórica-metodológica, resultando en un estado de indigencia analítica que se habría propagado en las ciencias sociales en Brasil.

Las preocupaciones de Fábio Wanderley son serias y pertinentes y, para el antropólogo, preocupantes. En la medida en que, en los últimos tiempos, ha crecido el prestigio y/o visibilidad de la antropología en el ámbito de las ciencias sociales en Brasil - prestigio y/o visibilidad que estaba lejos de tener hace veinte años atrás - se estableció, en el contexto de la ANPOCS², una visión

\footnotetext{
1 Fábio Wanderley Reis, 1991 - “O tabelão e a lupa: teoria, método generalizante e idiografia no contexto brasileiro". Revista Brasileira de Ciências Sociais n¹6, ano 6 :27-42.

${ }^{2}$ Asociación Nacional de Pos-graduación e Investigación en Ciencias
}

\section{Los antropólogos y sus linajes de Mariza Peirano}

Universidade de Brasilia, Brasil. E-mail: mpeirano@uol.com.br

\author{
Publicado originalmente como: Peirano, Mariza, 1991, \\ "Os antropólogos e suas linhagens". \\ Revista Brasileira de Ciências Sociais (16) 6: 43-50
}

Traducción: Malena Hopp y Sabina Regueiro CONICET, Universidad de Buenos Aires.

de que, en cuanto la sociología y la ciencia política se sientan en crisis, todo va bien con la antropología o con los antropólogos: la enseñanza es adecuada, los alumnos son bien formados teóricamente, la investigación de campo continúa siendo característica de la disciplina, cursos de graduación perfeccionan la formación uniendo investigación y enseñanza; en suma, la disciplina avanza.

\section{Generalización/universalización}

A diferencia de la ciencia política, la antropología no se ve como específicamente caracterizada por la disposición nomológica, sistemática y generalizante del estudio de las leyes sociales - disposición anunciada por Fabio Wanderley - ni tampoco como un tipo de sociología que, por sus características generales, se aplica a diferentes contextos, esto es, cuyos métodos e intereses son comunes a la ciencia social como un todo. Notoriamente preocupada por la especificidad del objeto de investigación, la antropología tal vez sea entre las ciencias sociales, paradojalmente la más artesanal y la más ambiciosa: al someter conceptos preestablecidos a la experiencia de contextos diferentes y particulares, ella procura disecar y examinar, para entonces analizar, la adecuación de tales conceptos. Para algunos, la antropología ha sido, por eso, teóricamente parasitaria, apenas probando las generalizaciones frecuentemente etnocéntricas de otras disciplinas en base a casos investigados a través del método etnográfico. Para otros, ella fue la ciencia social de lo "primitivo" que, habiendo visto su objeto en extinción, avanzó en las áreas de interés de otras ciencias. El objetivo más general, aún así, fue siempre la búsqueda de una visión alternativa, más genuina tal vez, de la universalidad de los conceptos sociológicos. Por lo tanto, no son grandes teorías ni abarcativos marcos teóricos los que la informan/conforman (aunque el estructuralismo haya sido la gran teoría social de del siglo XX) sino que al contrastar nuestros conceptos con otros conceptos nativos, ella se propone formular una idea de humanidad construida por las diferencias.

Todo comenzó con el desafío que Malinowski introdujo al confrontar, por un lado, trobriandeses de carne y hueso, y por otro, las grandes teorías evolucionistas de

Sociales (Nota de las traductoras). 
comienzos del siglo XX. Mitificado por haber introducido la investigación de campo intensiva, conocido por su obsesión por el native's point of view, criticado por el funcionalismo que un día creyó haber inaugurado, Malinowski tal vez haya desempeñado un papel aún más importante que todas esas proezas. Es que a él le cupo confrontar las teorías sociológicas, antropológicas, económicas y lingüísticas de la época con las ideas que los trobiandeses tenían con respecto a lo que hacían. Es más: al confrontar tales ideas con sus propias observaciones in loco, pudo percibir que había "residuos" no explicados: el Kula - el intercambio ceremonial de conchas de spondylus rojos por brazaletes de conchas blancas entre determinados socios en el extenso círculo de islas en el extremo oriental de Nueva Guinea - no era apenas un fenómeno meramente económico de intercambio de bienes preciosos, sino que abarcaba las esferas de lo religioso, de la política, de la mitología, de los ritos, repercutía en las formas lingüísticas e incluía también el comercio puro y simple, creando un círculo cerrado de relaciones entre las islas del archipiélago. Ese verdadero descubrimiento, resultado de la confrontación entre la teoría (y, por qué no, del sentido común europeo de la época) y la observación de los nativos melanesios, tal vez explique por qué volvemos constantemente a esa experiencia para develar la peculiaridad del quehacer antropológico. La lectura obligatoria de Malinowski (y de otros "héroes fundadores") se explica, así, especialmente por su carácter ejemplar de descubrimiento antropológico. En este sentido, importa menos la validez de sus propuestas (muchas consideradas superadas) que la vigencia de las teorías sobre magia, mitología, lenguaje, etc. que no son totalmente de Malinowski, sino el resultado del encuentro de Malinowski con los trobriandeses.

Aún así, se puede argumentar, que el feedback entre investigación y teoría constituye el procedimiento básico del conocimiento científico en general y que, para todas las ciencias humanas, no existe hecho social independiente de la referencia a la totalidad de la cual forma parte. Sin embargo, la investigación de campo antropológica, concebida como la búsqueda incesante del diálogo con el "otro", amplía y torna más explícitos esos presupuestos. Así, el extrañamiento pasa a ser no sólo una vía por la cual se da la confrontación entre varias "teorías", sino también un medio de autorreflexión. La reflexión sobre las ideas nativas muestra, por ejemplo, cómo el concepto de estratificación social es inadecuado en el contexto de castas de la India - y, si es pobre para explicar el mundo indiano, consecuentemente es limitado también para el mundo occidental -, o cómo la idea de tiempo, vivida en un lenguaje espacial entre los Illongot de las Filipinas, puede hacernos repensar nuestros propios conceptos nativos. Por otro lado, como el observador es parte integrante del proceso de conocimiento y descubrimiento, se puede decir, como ya se hizo anteriormente, que en la antropología no existe hecho social, sino "hechos etnográficos", destacando que hubo selección en lo que fue observado e interpretación en el relato. Florestan Fernandes pensaba como un antropólogo cuando, en la década de 1950, fue despedido de una investigación dirigida por Donald Pierson por haber cuestionado la orientación hacia la explicitación anticipada de las hipótesis teóricas que orientarían el análisis de cada documento etnográfico. Aislar un hecho antes de llegar al significado de la totalidad del contexto empírico era violencia para Florestan, visión por la cual pagó, al ser blanco de otra violencia.

En un abuso analógico, podríamos pensar entonces que, aunque la temática de la disciplina sea básicamente durkheimiana, la inspiración metodológica viene de Weber. O incluso, al focalizar el juego de espejos que la observación de la diferencia produce en el cuadro conceptual establecido, reconocer allí la tensión entre el iluminismo en el cual la disciplina fue fundada y el romanticismo alemán, en el cual se inspiró. Metáforas a parte, si bien una investigación etnográfica se realiza con el objetivo de desafiar conceptos establecidos e incluso la investigación de campo es característica de la disciplina, ese no es el objetivo final del antropólogo. Ya se ha dicho que la antropología estudia problemas y no pueblos (Evans-Pritchard, 1950) y, más recientemente, que los antropólogos no estudian aldeas, sino en aldeas (Geertz, 1973). Pero el hecho es que, aunque el coyunturalismo etnográfico apunte a una reflexión teórica, son las monografías lo valioso que conserva la disciplina. La razón es obvia: fue el kula de Malinowski el que le permitió a Marcel Mauss concebir el "hecho social total" y ayudó a Karl Polanyi a discernir la "gran transformación" en Occidente. Las observaciones de Evans-Pritchard (1940) sobre los Nuer descentralizados, las de Geertz (1980) sobre el theater-state en Bali, las de Stanley Tambiah (1976) sobre la galactic polity asiática, o las de Edmund Leach (1954) sobre la alternancia gumsa - gumlao en Alta Birmania, naturalmente traen consecuencias para la concepción sociológica del sistema político. De la misma manera, Louis Dumont (1980) fue a la India a descubrir la ideología individualista en el renunciador y, a la vuelta, detectó la perversión de la noción de jerarquía en el racismo occidental. Aquí, nos internamos junto a grupos tribales para descubrir cosmologías tanto o más sofisticadas que las producidas por pensadores occidentales; o junto a poblaciones campesinas para percibir relaciones sociales que están basadas más en una ética de la honra, jerarquía y reciprocidad que en nuestros principios de lucro y ganancia (éstos, sí, engendrados por la "gran transformación" por la cual el campesinado no pasa necesariamente) ${ }^{3}$. La observación de un pequeño frente de expansión en Marabá llevó a Otávio Velho (1976) a teorizar sobre el capitalismo autoritario en Brasil y, años después, basado en los mismos datos empíricos, a relacionar las categorías locales del bien

\footnotetext{
${ }^{3}$ Ver, por ejemplo, los trabajos de Eduardo Viveiros de Castro, en el área indígena, y de Klaas Woortmann, sobre el campesinado.
} 
y del mal con la teología cristiana (1987). En suma, el progreso de la antropología consiste en sustituir poco a poco los conceptos (sociológicos o no) por otros, más adecuados por ser más abarcativos, más universales o, en palabras de Louis Dumont, "más libres de sus orígenes modernos". Occidente se torna, entonces, una entre varias posibilidades de realización de la humanidad.

Así, queda claro que la etnografía no es algo que se hace espontáneamente, ni que se pueda prescindir de la inclinación o el talento. Pero las meras descripciones de un fenómeno de una cultura en términos de otra, son imitaciones necesariamente pobres de la práctica antropológica y, por definición, están condenadas a no pasar de afirmaciones de tipo populachero, aquello que Fábio Wanderley llama de estilo "periodístico" que invade las ciencias sociales. Si, entonces, las observaciones son realizadas no sólo para describir lo curioso, lo exótico o lo diferente por si mismos (por el natural interés que despiertan), sino también y principalmente para universalizarlas, estas dos direcciones - la especificidad del caso concreto y el carácter universalista de su manifestación - llevan a un proceso de refinamiento de problemas y conceptos y no, como propalan los estereotipos con respecto al empirismo de la antropología, a un cúmulo de informaciones sobre situaciones bizarras o, cuando falta talento, a "largas deposiciones en estado bruto de mujeres de la periferia urbana". Penosos para todos los cientistas sociales, tal vez sean equivalentes a las necesarias, aunque no menos tediosas, descripciones de tablas estadísticas, por ejemplo. La compilación de las posibilidades humanas Ilevaría naturalmente apenas a leyes y a reglas sociales - objetivo que los antropólogos descartan, luego de mucha polémica, a partir de los años 1960 - o a una contemplación estética de las diferencias en oposición al objetivo de largo alcance de alcanzar un universal modificado.

\section{Teoría e historia de la antropología}

Este, entonces, es el tronco del problema: si en la antropología la creatividad nace de la relación entre la investigación empírica y los fundamentos de la disciplina, entonces la investigación de campo surge como algo más que un mero ritual de iniciación en el cual el antropólogo prueba que "sufrió pero resistió". La soledad, aunque buena compañera de los descubrimientos de la alteridad, no es el camino virtuoso y mágico que, por si solo, produce buena antropología. Más allá del hecho de que la distancia necesaria para producir el extrañamiento pueda ser geográfica, de clase, de etnia u otra, ésta será siempre psíquica, los conceptos nativos requieren, necesariamente, la otra punta de la corriente, aquella que liga al antropólogo a los conceptos propios de la disciplina, esto es, a la tradición teórico-etnográfica acumulada.

Es por eso, tal vez, que habiendo llegado a los cursos de teoría antropológica pensando que iban a encontrar autores capaces de ofrecer la fórmula del buen trabajo, los alumnos descubren, para su sorpresa o desaliento, aunque frecuentemente como desafío, que la teoría antropológica es teoría e historia de la antropología, de la misma forma que es teoría y etnografía. Es sobre la tensión entre el presente teórico y la historia de la disciplina que la tradición de la antropología se transmite, resultando que, en el proceso de formación, cada principiante establece su propio linaje como inspiración, de acuerdo con las preferencias que son teóricas pero también existenciales, políticas, a veces estéticas e incluso de personalidad. Así, más allá de los clásicos Durkheim, Marx y Weber, que enseñarán la postura sociológica, el antropólogo en formación entra en contacto con un verdadero árbol genealógico de autores consagrados (y otros malditos), en el cual construirá un linaje específico sin desconocer la existencia de otros. En la antropología, los linajes disciplinares son tan importantes que se pude imaginar que, sin ellos, el antropólogo no tiene lugar en la comunidad de especialistas. Pero, como ocurre hasta en los más rígidos linajes africanos, los cambios son aceptados y, en este caso, vistos como "conversión". Este fue el caso de Marshall Sahlins que, partiendo de una vertiente economista - ecológica, se convirtió al estructuralismo, como lo demuestran los cambios de Stone age economics (1972) a Cultura y razón práctica (1979) o Islas de historia (1990).

En ese proceso de pasaje disciplinar, el conocimiento etnográfico con respecto a varias sociedades y culturas se enriquece. Eso significa que un antropólogo bien formado teóricamente es un antropólogo bien informado etnográficamente. Para algunos, este entrenamiento a través de la literatura permite que, hoy, el antropólogo prescinda de la investigación de campo en sociedades desconocidas antes de confrontar la suya propia; para otros, se trata de la sorpresa de descubrirse súbitamente con capacidades inesperadas, como la de reconocer las diferencias estéticas entre una máscara latmul de Nueva Guinea, de otra de los Kwakiutl del Noroeste de América del Norte, o de los Bororo del Brasil Central, a través de la lectura de Bateson, Boas y Lévi-Strauss. Pero el hecho más sobresaliente tal vez sea el siguiente: la transmisión de conocimiento y la formación de nuevos especialistas - a través de los procesos por los cuales se dio el refinamiento de conceptos, pero se mantuvieron los problemas favorece una práctica en la cual los autores nunca son propiamente superados. Nombres conocidos, que un día fueron criticados y combatidos, frecuentemente son incorporados en las generaciones siguientes porque, releídos, revelan riquezas antes desconocidas. Este mecanismo de incorporación de autores, que marca la disciplina, tal vez se explique como un culto a los ancestros: aunque raramente se encuentra hoy un especialista que se autodefina como un estructuralista stricto sensu, también difícilmente un antropólogo deje de incluir varios de los principios del estructuralismo en 
su práctica disciplinar. Lo mismo tal vez, pueda ser dicho con respecto a todos los fundadores de linajes, en un mecanismo que no respeta fronteras: aquí en Brasil, Darcy Ribeiro incorporó a Herbert Baldus, que fue incorporado, junto con Florestan Fernandes, por Roberto Cardoso de Oliveira, y así sucesivamente (El reconocimiento de las filiaciones es, aún así, mucho menos explicitado que en el caso de las vinculaciones extranjeras).

Visto bajo esta óptica, el "coyunturalismo" que Fábio Wanderley Reis apunta en la inspiración antropológica es propia de aquellos que se limitan apenas al inmediatismo de la experiencia, desde un lugar de extrañamiento y relativización, y que se olvidan de la tradición de la disciplina: si las diversas coyunturas con las que el antropólogo se enfrenta lo hacen repensar la teoría sociológica clásica occidental, esto - que es la fuerza de la antropología - puede tornarse su debilidad, si apenas lo exótico es privilegiado. En Brasil, el efecto pernicioso aumenta cuando, bajo un pretendido compromiso político, intentamos extrañar y rescatar camadas oprimidas de la población y acabamos muchas veces haciéndoles el disfavor de revelarlas, por ejemplo, en nuestros relatos tediosos sobre las mujeres de la periferia urbana. Volveré sobre este asunto.

\section{Consecuencias}

Las observaciones anteriores tienen por lo menos tres consecuencias inmediatas. Primera: no hay propiamente una forma de enseñar a hacer investigación de campo. Esta es una conclusión antigua; no sólo de profesores bien intencionados, sino también de estudiantes interesados, aunque atónitos. La experiencia de campo depende, entre otras cosas, de la biografía del investigador, de las opciones teóricas dentro de la disciplina, del contexto socio-histórico más amplio y, no menos, de las situaciones imprevisibles que se configuran en el día a día, en el lugar donde se realiza el trabajo de campo, entre investigador e investigados. Esta es, tal vez, la razón por la cual los proyectos de investigación de los estudiantes de antropología siempre se tropiezan con la cuestión "metodología", cuando éstos compiten con colegas de otras ciencias sociales. Si bien es imposible anticipar las eventualidades que resonarán en la experiencia vivida del aquí y ahora, las teorías aprendidas de otros pueblos y otros tiempos, hacen que sea posible alertar al estudiante de los problemas corrientes con los que probablemente se enfrentará, porque otros ya los han enfrentado. En suma, en la antropología, el entrenamiento metodológico se hace mejor cuando se han incorporado las monografías clásicas o, lo que es lo mismo, cuando se ha derivado de los cursos teóricos.

Segunda: A pesar de la confianza en la excelencia de sus herramientas conceptuales, en su método de investigación de campo y en su tradición disciplinar, la antropología no se reproduce como una ciencia normal de paradigmas establecidos, sino por una manera determinada de integrar teoría e investigación, de modo de favorecer nuevos descubrimientos. Estos quedan sujetos a la posibilidad de que una investigación de campo pueda revelar, no al investigador, sino en el investigador, aquel residuo incomprensible, pero potencialmente revelador, que existe entre las categorías nativas presentadas por los informantes y la observación del etnógrafo, inexperto en la cultura estudiada y apenas familiarizado con la literatura teórico-etnográfica de la disciplina. Las impresiones del campo no son recibidas sólo por el intelecto; éstas tienen un impacto en la personalidad total del etnógrafo, haciendo que diferentes culturas se comuniquen en la experiencia singular de una sola persona ${ }^{4}$. Estas consideraciones tal vez expliquen dos cosas: por qué los antropólogos siempre consideran una instancia empírica específica y por qué en la investigación de campo, es común constatar que la vida imita a la teoría. En el primer caso, la búsqueda de lo específico, de lo diferente-donde tal vez se revele aquel "residuo" que permitirá el avance de la observación etnográfica y consecuentemente, la posibilidad de refinamiento teórico- pasa a ser una práctica regular de los antropólogos, que ya bautizaron estos casos como "incidentes reveladores" (Fernandez, 1990); en el segundo caso, es el investigador, entrenado tanto en los aspectos más extraños como en los más corrientes de la conducta humana, el que encuentra un ejemplo vivo en la literatura que lo informa.

Tercera: si la investigación-con-teoría define la empresa antropológica, entonces no hay lugar para una crisis en tanto haya investigaciones nuevas junto con las reflexiones teóricas correspondientes (y viceversa). La última crisis vivida por la antropología dataría de los años 1960, cuando los antropólogos, no habían percibido que, más que un determinado objeto de estudio concreto, la disciplina se dedicaba al análisis de las diferencias culturales. Sólo cuando la inminencia de la extinción de los grupos indígenas y el rechazo de la antropología por las ex-colonias africanas se tornaron reales -amenazando la propia continuidad de la disciplina- los antropólogos tomaron conciencia de que estaban equivocados al suponer que los llamados pueblos primitivos, definían la disciplina. En realidad, ellos nunca habían estudiado solamente los pueblos primitivos, pero al hacerlo, habían aprendido a reconocer las diferencias entre el mundo de los antropólogos y el mundo nativo, o como descubrió Lévi-Strauss en 1960, habían percibido los "desvíos diferenciales" entre culturas diferentes -éstas sí imposibles de desaparecer, a pesar de todos los ideales de la globalización. De ahí en adelante, por etapas, los nativos dejaron de ser apenas los "primitivos" y se transformaron en "otros", sucesivamente remotos en el espacio, remotos en el tiempo, menos remotos en la propia sociedad, hasta la conclusión reciente de que

\footnotetext{
4 Evans Pritchard (1972) y Louis Dumont (1970, p.157) discutieron el impacto de la investigación de campo en la personalidad del etnógrafo.
} 
"ahora somos todos nativos" de Geertz (1983), quien repite a Durkheim un siglo después. Es una posibilidad entonces, que los pronósticos siempre optimistas de los antropólogos que frecuentan las reuniones de la ANPOCS tengan origen, por lo menos en parte, en la consciencia de que el proceso complementario entre investigación de campo y teoría, resguarda a la antropología de crisis periódicas, más allá del hecho de que las diferencias que interesan a la disciplina nunca desaparecerán.

Pero es también posible que el ejemplo clásico de los Nuer de Sudán, estudiados por Evans Pritchard (1940), sea aplicable a la comunidad de antropólogos: sin un sistema político centralizado, los "anárquicos" Nuer se organizaban a través de la identificación de las comunidades locales con los linajes de un único clan, en un sistema fluido, en el cual la separación o la unión de los segmentos adyacentes de un mismo orden son expresadas en un cuadro de valores ligados a la descendencia: soy miembro del grupo $A$ en una situación que opone el grupo $A$ al B; en otra situación, en la cual dos segmentos de A están opuestos - digamos $A_{1}$ y $A_{2}$ - yo no me defino como miembro de $A$, pero sí de $A_{1}$, y así sucesivamente. El ejemplo de los Nuer, tal vez pueda explicar por qué los antropólogos, que viven oposiciones en el día a día, uniéndose o separándose según las comunidades locales, los linajes o la "descendencia", adoptan la postura de un sólido "grupo unilineal" en el contexto tribal más inclusivo de la ANPOCS.

\section{El modismo actual}

Todo este cuadro se complica un poco cuando se constata que un grupo de antropólogos norteamericanos, autodenominados reflexivos o pos-interpretativos, reconoce la existencia de una de aquellas crisis en la disciplina. En la medida en que el énfasis principal de ese grupo reside en el cuestionamiento de la investigación de campo como práctica, se pierde la tensión principal entre teoría e investigación -y la crisis se instala, confirmando la propuesta anterior. Infelizmente para nosotros, éste es el modismo actual. Como otras influencias externas, que en el primer momento no nos damos el trabajo de cuestionar para después incorporarlas a las tradiciones locales, ésta termina por transformarse en una aceptación perezosa.

En los Estados Unidos ese grupo, pequeño, pero activo, cuestiona la tradición de la investigación de campo en sus aspectos morales y epistemológicos: ellos que siempre buscaron lo exótico más allá del mar, se confrontan hoy con los problemas de las relaciones de poder y de dominación que tradicionalmente caracterizaron al trabajo de campo entre investigador e investigados. Empeñados en una autocrítica de esas relaciones y de la legitimidad de la investigación tradicional en un mundo que se considera pos-colonial, ellos proponen que el resultado de la investigación no sea fruto de la observación pura y simple, sino de un diálogo y de una negociación de puntos de vista (Marcus y Fischer, 1986). De ahí a la crítica de los estilos etnográficos, al cuestionamiento de la autoridad del texto, al descreimiento de las macro teorías, al énfasis en la fragmentación de la experiencia, a la imposibilidad de alcanzar generalizaciones o universalismos, la distancia es corta. Suspendida la cuestión teórica, las preocupaciones se focalizan en la auto-reflexión (de inspiración democrática) sobre el antropólogo en el campo y sobre el estilo retórico en el cual sus resultados serán comunicados. Esta postura, que frecuentemente se desliza hacia la auto-referencia, ya produjo la anécdota en la cual el investigador llega a un grupo indígena y después de mucho conversar, escucha del nativo la pregunta: "Ahora que ya hablamos dos horas sobre vos, ¿podemos hablar un poco sobre mi?"

En los Estados Unidos, esos cuestionamientos, hace unos años considerados experimentales, como todo lo que fascina, se rutinizaron. Hoy, Clifford Geertz, el mayor inspirador de esa vertiente, se hizo público buscando eliminar las exageraciones de un lado y del otro: en 1984 defendió el anti-anti-relativismo y, en 1988, con el mismo dedo amonestó al nuevo estilo como una dolencia endémica que, en vez de producir etnografías, produce diarios, reflexiones meta científicas, las Ilamadas "etnografías experienciales", periodismo cultural, activismo sociológico, todos informados por una sinceridad redentora que no pasa de una esperanza fútil y estéril.

Como ocurre con todo lo que es transplantado de forma acrítica en Brasil, esta tendencia también tiene efectos perniciosos (Trajano, 1987, Sena, 1987). Pero aquí el mayor problema no está en la calidad estética (que indirectamente, es teórica) de lo que se produce, sino principalmente en la trasmisión de la disciplina. Primero porque estimula la búsqueda de nuevos estilos en aquellos que todavía no se iniciaron propiamente en la disciplina, fortaleciendo la creencia de que la retórica sustituye la densidad teórica; segundo, y más grave, porque promueve un descrédito prematuro e inconsecuente de la tradición de la disciplina, haciendo que estudiantes mal informados pasen a ver en los textos clásicos ejemplos superados del realismo etnográfico, de autores positivistas que nunca prestaron la debida atención a la dimensión existencial del encuentro etnográfico. Algunos llegan a defender la triste "postura ecléctica", en la cual existe mayor tolerancia en relación con la incompletud de los sistemas teóricos. Frente a esta situación, detectada principalmente entre los estudiantes en la etapa de su tesis, no es extraño que muchos de nosotros pasemos a defender una actitud tradicional, para impedir que el bebé sea tirado junto con el agua de la bañera. Después de una larga historia en la que se procuró definir a la antropología como un tipo de conocimiento específico -y no faltarán aquellos que intentan definirla como "ciencia" (a través de abordajes como el "funcionalismo", el "estructural-funcionalismo", el "estructuralismo"), "arte", "traducción cultural", etc.-, 
hoy la "interpretación" parece echar mano de aquellos propósitos para transformarse en instrumento de ralos ideales humanistas.

Por otro lado, parece olvidarse que, en el caso brasilero, las cuestiones morales tienen una connotación eminentemente política, además de un origen diferente al que tienen en el caso norteamericano: hasta ahora, nuestros "otros" fueron básicamente buscados dentro de los límites de las fronteras nacionales y, ciertamente cuando éstas fueron traspasadas, las cuestiones de dominación que estuvieron presentes en las relaciones entre ingleses y africanos, norteamericanos y asiáticos, franceses y árabes norteafricanos, no serán equivalentes a las que iremos a mantener con India, Guiné Bissau, Argentina o Estados Unidos. Resta entonces, la esperanza de que asimilado lo que tiene de positivo, y pulidos los excesos, la antropología interpretativa nos pueda ayudar a volver a la tradición, para avanzar.

\section{Diagnóstico}

Si: (a) la investigación de campo y la tradición teórica de la antropología se relacionan en el día a día de los especialistas, (b) la investigación de campo, concebida como el encuentro con un "otro" es constitutiva del conocimiento disciplinar, (c) la teoría antropológica se desenvuelve pegada al conocimiento etnográfico, (d) teoría e historia de la antropología son inseparables, tal vez se puedan detectar algunos puntos de estrangulamiento, en los cuales mala hechura y mala lectura ocurren y afectan negativamente a la propia antropología y a las disciplinas afines. Veamos.

Primero, en relación con la formación teórica. Es preciso remarcar que los modismos seducen tanto a alumnos como a profesores y que la indolencia didáctica encuentra respaldo en el letargo de los alumnos, cuando se trata de formar nuevas generaciones. Aunque se lea mucha teoría (esto es filosofía y epistemología), se lee poca teoría antropológica. Los cursos de teoría antropológica, son por definición arduos y largos e incluyen, necesariamente la lectura de monografías clásicas en su totalidad. Si es verdad que el estilo etnográfico contiene en si mismo elementos teórico-metodológicos, son las monografías, construidas dentro de innumerables posibilidades, las que dejan vislumbrar el curso intelectual del investigador, que permiten situarlo en un determinado contexto disciplinar, y más importante, las que hacen justicia al autor porque, dándole la palabra, permiten eventualmente "redescubrir" en ellos una riqueza inesperada. En otras palabras, meros trechos de monografías no bastan.

Segundo, en relación a la investigación. Aquí el problema mayor tal vez esté en el hecho de que, salvo raras y felices excepciones, las nuevas generaciones no siempre trasponen barreras significativas. Aunque la distancia geográfica haya sido tradicionalmente garantía de alteridad, y aunque la opinión de los especialistas sea que el estudio de la propia sociedad es tarea de los investigadores más experimentados, la cuestión es más compleja. Aquí se trata de la opción de buscar al "otro" cerca de casa, no sólo como resultado de una limitación de recursos (que no convence totalmente) sino como resultado, en Brasil, de una tradición política oriunda de la matriz de la sociología (de la cual nacemos de una costilla) que privilegia el estudio de grupos oprimidos de la sociedad o de minorías étnicas, sociales, regionales, etc. No es necesario llamar la atención sobre el hecho de que la relevancia teórica no se confunde con la relevancia política, así como los problemas epistemológicos no equivalen a los problemas de clase. De esta inadecuación entre teoría y objeto de estudio, los resultados negativos surgen a veces de los excesos teóricos o, más frecuentemente, de una investigación frágil y sin densidad.

Ejemplos de mala hechura, no deben oscurecer, con todo, los proyectos más consolidados que se ramifican en varios programas de pos-graduación en el país y que son frecuentemente enriquecidos por perspectivas teóricas diversas, sobre temas relacionados con grupos indígenas (de cosmología a contacto interétnico), con el campesinado (de luchas sociales a ética y moralidad), las investigaciones sobre el campo intelectual, los relevamientos socio-antropológicos de las grandes metrópolis, sin hablar de los emparentados estudios sobre el proletariado, periferia urbana, clases medias y elites, o cuestiones de identidad religiosa, cívica, regional, entre otros (definidos temáticamente, los recortes teóricos producirían otros agrupamientos).

Tercero, en relación con los límites de nuestro mundo académico. Aquí cabe marcar que lo que se dice respecto del locus de la investigación, algunas investigaciones ya traspasan las fronteras nacionales, con estudiantes yendo a Guiné Bissau, Costa Rica, Goa, Argentina, Estados Unidos. Ellos seguramente traerán una dimensión contrastante enriquecedora. En relación con los interlocutores intelectuales, tal vez tengamos que convivir con el problema de consumir la última moda internacional en francés y en inglés, para debatirla en el portugués local. Este hecho crea una situación sui generis: aislados del universo académico que se define como internacional, podemos darnos el lujo de incorporar ideas sin la carga de dar cuenta de los resultados obtenidos. Por otro lado, perdemos la visibilidad que podríamos alcanzar, así como, más importante aún, la validación y el diálogo externo que podría darnos una mejor dimensión de nuestro quehacer. Además de eso, fascinados por los modismos, dejamos de lado trabajos competentes, pero "tradicionales", tanto aquí como afuera.

Finalmente, cabe volver a la especificidad del caso brasilero en relación con la vocación "pluralista" de las ciencias sociales, como apunta Antônio Cándido, 
dominaba las primeras generaciones que se formaron en los años 1950 en San Pablo, y que después dio lugar a la división del trabajo intelectual con la que hoy convivimos. Ese pluralismo saludable, que hacía que se cuestionara si un determinado trabajo era sociología o antropología, cine o teatro, sociología o crítica, (y que permitía que un antropólogo se tornara sociólogo; un sociólogo crítico literario; y un filósofo antropólogo), resuena hoy en la ANPOCS, heredera de esa tradición y tal vez el fuero más legítimo para un interdisciplinariedad intelectual que no se presenta como modismo, no es impuesta, pero siendo agradable e histórica, debe ser concientemente preservada con sus difíciles implicaciones, a pesar de las diferencias institucionales inevitables. Esta fue la motivación de este trabajo.

\section{Bibliografía}

Dumont, L. (1970) Religion, politics and history in India. Paris, Mouton.

Dumont, L. (1980) Homo hierarchicus. Chicago University Press.

Evans Pritchard, E. E (1940) The Nuer, Oxford University Press.

Evans Pritchard, E. E (1972) Social anthropology. Londres. Routledge \& Kegan Paul.

Fernandez, J. (1990) Tolerance in a repugnant world, Ethos, vol. $18, \mathrm{n}^{\circ} 2$

Geertz, C. (1973) The interpretation of cultures. Nueva York, Basic Books.

Geertz, C. (1980) Negara. The theater state in Bali. Princeton, University Press.

Geertz, C. (1983) The way we think now, in Local Knowledge, Nueva York, Basic Books.

Geertz, C. (1984) Anti-anti-relativism, American Anthropologist, vol. 86, $\mathrm{n}^{\circ} 2$, junho. Traducido al portugués por André Villalobos y publicado en RBCS vol. 3, n 8, pp. 5-19.

Geertz, C. (1988) Works and lives. The anthropologist as author. Standford University Press.

Leach, E. (1954) Political systems of highland Burma. Nueva Jersey, The Athlone Press.

Leach, E. (1961) Rethinking anthropology. Nueva Jersey, The Athlone Press.

Marcus, G. y Fischer, M. (1986), Anthropology as cultural critique. Chicago University Press.

Peirano, M. (1990), O pluralismo de Antônio Cândido. Revista Brasileira de Ciências Sociais, vol. 5, n 12.

Sahlins, M. (1972) Stone age economics. Chicago, Aldine Publishing Company.

Sahlins, M. (1979), Cultura e razão prática. Rio de Janeiro, Zahar. Primeira edição norteamericana, 1976, Chicago University Press.

Sahlins, M. (1990), Ilhas da história. Rio de Janeiro, Jorge Zahar Editor.

Sena, S. (1987), Em favor da tradição, ou falar é fácil, fazer é que são elas, Série Antropologia n 53 . Universidade de Brasilia.

Tambiah, S. (1976), World conquerer and world renouncer. Cambridge University Press.

Trajano, W. (1987), Que barulho é esse, o dos pósmodernos?, Anuário Antropológico 86, p. 133-151. Tempo Brasileiro/Universidade de Brasilia.

Velho, O. (1976), Capitalismo autoritário e campesinato. São Paulo, Difel.

Velho, O. (1987), O cativeiro da besta fera, Religião $e$ Sociedade, vol. 1, n 14, pp. 4-27. 
